\title{
Usability Assessment of the Electronic Patient Management System for AIDS \& TB Services in Zimbabwe
}

\author{
Takunda Mukumba, Yashik Singh \\ Department of Telehealth, School of Nursing and Public Health, College of Health Sciences, University of \\ KwaZulu-Natal, KwaZulu-Natal, South Africa \\ Email: tkndmukumba@yahoo.co.uk
}

Received 9 September 2014; revised 25 October 2014; accepted 26 November 2014

Copyright (C) 2014 by authors and OALib.

This work is licensed under the Creative Commons Attribution International License (CC BY).

http://creativecommons.org/licenses/by/4.0/

(c) $\underset{\mathrm{EY}}{0}$ Open Access

\section{Abstract}

Introduction: Medical care is provided by multidisciplinary teams. There is great need for high levels of coordination among teams. Paper medical records pile up leading to logistical difficulties owing to the passive nature of paper records. Sometimes finding a piece of medical history can be difficult. Information and communication technology solutions can solve such problems. The Zimbabwean AIDS \& TB Unit under the Ministry of Health installed an electronic Patient Management System in 83 health facilities to attempt to alleviate some of the disadvantages of the paper system. It is important for the Unit to carry out a usability assessment of the system, to determine if it is appropriate and how usable it is. The more competently the users operate the system, the better the service AIDS \& TB patients receive from them. Aim: To perform a usability assessment of the new electronic Patient Management System based on user experiences at the ministry of health's 83 high volume facilities. Methods: A cross-sectional survey of the 83 facilities was done using a self-administered questionnaire as the data collection tool. Multiple linear regression analysis was done to investigate factors affecting usability of the system. Differences between dgemographic subgroups as well as socio-cultural subgroups were investigated along with differences between work experience backgrounds and how they affected usability. Results: 48 (57\%) of the 83 facilities responded. In a multiple linear regression analysis only amount of time a facility had used the system was found to have a linear relationship with the usability of the system $\left(R^{2}=0.2623 ; 95 \%\right.$ CI). There were no differences between demographic subgroups as well socio-cultural subgroups. Chi $^{2}$ test between gender and usability gave $\mathbf{P}=0.3$ along with respondent's post. Discussion: The training that came with the installation of the system could have cancelled out some of demographic and socio-cultural factors that would have influenced the usability of the system since usability only varied with time. Acquiescence response bias and social desirability bias may be contributing factors. Conclusion and Recommendations: The perception of system usability and competency in use will improve with time. An assessment that captures all the facilities may give better insights into the issue of usability. 


\title{
Keywords
}

\section{Electronic Patient Management System (ePMS), Usability, Heuristics}

\author{
Subject Areas: Nursing, Public Health
}

\section{Introduction}

Medical care is provided in a highly coordinated environment consisting of complex work processes performed by multidisciplinary teams. There is often great need for high levels of coordination between these teams. Paper work often piles up in the form of medical records that often need referring back to. Sometimes finding a piece of medical history can be difficult. The paper based records may be misplaced, may not be legible enough to read or could have been destroyed by a natural disaster. This may lead to errors such as prescribing certain drugs to a patient who is allergic to them, since paper based records are passive. Coordination between these multidisciplinary teams can be flawed such that patients may have the same medical procedures unnecessarily repeated or done incorrectly.

Using information and communication technology solutions can help solve such problems. An example is electronic medical records. In Zimbabwe, the AIDS \& TB Unit of the Ministry of Health and Child Care (MoHCC) installed an electronic patient management system (ePMS) in what they deemed were high volume (health) facilities in order to alleviate some of the problems of the overburdened paper based system. Computers are not as passive as paper, and can give feedback when tasks are being performed. An alert signal when you prescribe a drug that a patient is allergic to for example. Medical records can also be stored in legible form as computer based records. The use of computer data backups can also help protect records from destruction and loss.

\section{The Ministry of Health and Child Care (MoHCC) Case of the AIDS and TB Unit}

HIV prevention programmes in Zimbabwe began the 1990's. Since then patient data has been collected and processed manually using paper based systems. However, because of the large numbers of people requiring HIV services, these paper based systems have become over-burdened. This has negatively affected the quality and timeliness of data used to monitor, track and report on patients [1].

In order to solve this problem MoHCC's AIDS and TB Unit has been installing electronic patient management systems (ePMS) in health facilities offering HIV services and prevention programmes. In the period between December 2012 and December 2013, MoHCC completed its initial phase of ePMS rollout in 83 "high volume" facilities. The intention is to expand the installation to 350 health facilities in the $2^{\text {nd }}$ year and up to 454 health facilities by the $4^{\text {th }}$ year [1].

The AIDS \& TB Unit is carried out assessments of their ePMS based on the first phase of their ePMS installation in order to correct the short comings of the first phase of ePMS installation and to prevent passing them on the next phases of the ePMS roll out.

The AIDS and TB Unit therefore sent its teams to the different facilities scattered throughout the provinces of Zimbabwe to carry out it assessments and investigations on the ePMS. This study was piggybacked on one of the ePMS team's deployment to ePMS facilities and utilized that opportunity to distribute self administered questionnaires to ePMS users at the sites that the ePMS teams were visiting. Thus carrying out the study: Usability assessment of the electronic patient management system for AIDS and TB services in Zimbabwe.

The usability assessment was important for the purposes of investigating the perceptions and experiences of the users of the system, in order determine whether or not the users of the system are comfortable with system. The more comfortable the users are with the system the better they can use it and the more benefits patients can get from an improved and more efficient service delivery system. Usability is a quality attribute that assesses how easy user interfaces are to use [2]. This study will inform MoHCC's future decisions on how to proceed with the $2^{\text {nd }}, 3^{\text {rd }}$ and $4^{\text {th }}$ phases of its ePMS rollout and better maximize the benefits of ePMS by correcting the areas to do with usability (if any) that the users are having problems with. 
Usability testing is necessary because it gives a good indicator of how well the users of the ePMS system will be able to utilize it. Usability looks at the graphic user interface (heuristics) of the software and how it has been designed to be user friendly among other things. Usability aspects to do with the graphic user interface are known as heuristics and include software-user interaction, learnability, cognition facilitation, user control and software flexibility, system real world match, graphic design, navigation and exiting, consistency, defaults, software system interaction, help \& documentation and error management [2]-[4]. Use case frameworks have been designed to guide usability assessments based on these heuristics [4].

The AIDS and TB Unit needed a usability assessment of its electronic patient management system. Such information is crucial for the next phases of the system's roll out. Therefore, the purpose of this research was to perform a usability assessment of the ePMS, based on the users' perceptions and experiences, at the 83 high volume health facilities where the ePMS was installed in the $1^{\text {st }}$ phase of installation.

The specific objectives of this research were: To construct a tool/questionnaire to investigate the usability of the ePMS at the 83 high volume facilities offering AIDS and TB services; To apply the assessment tool on the 83 high volume facilities and; To investigate factors that affecting the usability of the ePMS.

\section{Usability Literature and Benetfits of ICT in Healthcare}

Information and communication technology (ICT) has evolved rapidly and has made significant contributions in many sectors if not all. The health sector is also benefiting from the adoption of ICT applications designed to meet this sector's needs. These include clinical decision support systems, electronic medical records, telemedicine systems, veterinary information systems, drug stock management systems, body area networks, tele-presnence and tele-robotic surgery among others.

Electronic Medical Record (EMR) systems are some of the ICT applications becoming popular in the health sector. In particular, they benefit hospitals, clinics and private practice doctors. Other health related organizations can also use EMR. Health insurers are one example. EMR is intended to keep track with a patient's health and medical history in an electronic format (Torrey 2012). Keeping these records in digital or computerized format makes them easy to retrieve. It also makes the patient's sequence of events within a health facility more efficient than one with paper records since relevant data is generally easier to retrieve. Different institutions will give different names to EMRs depending on the specific purpose for which they have them. Other forms of EMR are called electronic health records (EHR), personal health records (PHR) and electronic patient management systems (ePMS) among others.

EMR contains many components which include the following; computerized orders for prescriptions, computerized orders for laboratory tests, reports of the test results, patient support, clinical decision support systems, access to knowledge resources, billing and physician's notes, among others [5]. The ePMS mentioned in this study is essentially EMR. MoHCC calls it ePMS. Through the use of EMR, the information that was on patient paper charts is now kept electronically. This in turn solves issues to do with overburdened paper systems.

\subsection{Benefits MoHCC Can Derive from Using EMR}

EMR facilitates efficient administrative processes. Administrators skip the laborious process of having to dig through heaps of files to find patient information. EMR reduces cognitive load on the users by making patient information available at the click of a button. The patient goes through the hospital administrative processes faster and also gets treatment more expediently as a result.

Since ICT can duplicate information rapidly, once patient information is stored electronically, easy loss of that information can be prevented. Backup files can be made. Some EMR systems can automatically backup information. Fears of losing information due to natural disasters for example, are reduced. In the United Sates, after hurricane Katrina struck, patients who had their medical history in EMR systems got treatment easier, faster and safer since their accurate medical history was quickly retrieved and used [6].

Paper records will require large amounts of physical space to be stored as they accumulate. On the other hand EMR exists in electronic format and are stored in computers and servers which take up less physical space. Space that is taken up by medical records rooms and departments can be used for other purposes that are more clinical and hence the hospital can increase service delivery to its people through efficient use of space. The cost per square meter of building space is reduced since more of the building can now be used for more productive and/or income generating purposes. 
Medication errors are reduced when using EMR systems. Paper is passive; therefore it will not warn a health professional if he/she attempts to prescribe medication that a patient is allergic to. Electronic systems on the other hand are interactive and can prompt the user to follow the right steps for whatever procedure they are doing. Also, because all the hospital visits of a patient can be recorded in the EMR of patients together with the activities that took place, medical efforts are no longer duplicated. Clinicians can go straight to what has not yet been done for a patient since they can see all that was done for the patient on the EMR.

Diseases and disease trends can be monitored and tracked much more easily and possibly in real-time when using EMR since all the information can be obtained at the press of a button, complete and accurate. It is also easy to identify patients that are due for preventative measures for example patients that are at risk of drug resistance. The AIDS \& TB Unit (Zimbabwe) can better monitor early warning signs for HIV drug resistance, which is the case with the ePMS that they have installed in their health facilities.

When using EMRs, research is also easier to carry out. The results of the research tend to be more accurate from EMR than from paper records. This is because paper records are more prone to deterioration. Therefore not all information will be retrieved from paper records. Paper records can also be lost or misplaced, making it difficult to find all the information. Paper records also slow down research since researchers have to dig through heaps of paper files to find what they are looking for.

These are some of the benefits of electronic medical records among others.

\subsection{Usability and Heuristics}

Medical care is given in a highly coordinated and punctuated environment consisting of complex work process performed by multidisciplinary teams. This environment is almost always site and provider specific in terms of the workflow, the cognitive requirements and the decision making processes. ePMS or EMR systems are required to be effective in such clinical settings. The complex clinical environment is difficult to replicate in the lab where the ePMS is developed. Furthermore, even direct involvement of clinicians in the product development sometimes fails to accurately reflect clinician workflow [4].

In order to realize the full potential of the of the ePMS-user-patient relationship, wise choices must be made in terms of the choices of the system that a country or institution chooses to use. Issues such as environmental limitations and the uncertainty of the future need to be factored in. The information design of the system must be given careful consideration even after the implementation of a system so that the appropriate adjustments can be made [7].

Information design represents the art and science of preparing and conveying information so that it can be used by human beings with efficiency and effectiveness [7]. When done well it maximizes usability of information systems. An ePMS for example, should reduce the burdens of the following workloads on the user, among others; ergonomic workload, cognitive workload (to do with reading, thinking and deciding) and data comprehension [4]. This is achieved by providing the user with memory aid, computational aid, decision support and collaboration aid. The design of the system and the proper use of graphics within the user interface help achieve all this.

The system must use a language that the users are familiar with. The icons on the graphic user interface must be easy to understand in order to reduce cognitive workload. There should not be too much information-clutteron one page such that the user will struggle to find the appropriate information. Information required to perform a single task must be found on the same page and the system should not require the user to enter the same information over and over again. The system should provide task oriented action options such as ordering lab tests and electronic prescribing. Computational functions should be available on the software and graphs shown should aid data comprehension. The computer displays must force the user to view the data spatially rather than sequentially in order to aid comprehension. This is done by increasing data density, which is the number of entries in the data matrix/area of the graphic [8].

\subsection{Possible Factors Affecting Usability}

The needs of the site (or health facility) and the users will vary depending on many factors, particularly demographic, geographical and socio-economic factors. This also means that, how different users rate the usability of the same ePMS may vary.

Not everyone will find the same system usable, or rather, usable to the same extent. In some settings, for ex- 
ample, young people generally know how to use technological gadgets far much better than (older people) their parents. In other words young people generally find technological gadgets more usable than their parents do. The things that motivate youths to learn how to use gadgets are different from those that motivate the elderly to learn how to use the same gadgets. The enthusiasm of the 2 groups is different as and so is the willingness to learn how to use these gadgets. The young are fundamentally different from the old in terms of their technological abilities, communication skills and the way they are socialized [9]. Nasi and colleagues did a study in Finland, in his article he mentions that young adults aged between 16 - 34 years are by far the most active users of ICT. Social networks motivate young adults to use ICT more often than older age groups do. In a health facility, young adult employees are likely to learn work related ICT interventions faster than older employees and find ICT applications to be more usable. This is because of their frequent use of ICT outside of the work place. ICT applications in the work place may not feel like a new concept to them. This "age digital divide" is likely to influence variations in how employees at the same institution find their ePMS system.

Gender may also affect how usable one may find an ICT application such as an ePMS. Variations are likely to arise among employees in the same institution, using the same ePMS system. The ways in which ICTs are used by men and women reflect the sociocultural and economic contexts within which they are used [10]. This means in a health facility where both man and women are using the same ePMS and have the same opportunities to do so, their usability ratings are unlikely to be the same. It is the circumstance before the ePMS was introduced that may affect how usable an individual will found it. Gender disparities in ICT exposure, if they exist outside of the work place are likely to influence how people of different genders will feel comfortable using the new ICT application in the work place. If it is not a socio-cultural norm for example for a certain gender to use ICTs outside of the work environment, when ICTs are finally introduced in the work environment, people of that gender may take longer to adjust to the change.

Another factor that may cause variations in how users find the ePMS system usable is geographic location. If the ICT penetration in an area is low, that means for people in that area, regardless of gender, use of ICTs may not be a socio-cultural norm. Therefore if an ePMS is introduced in a health facility in such an area, they are likely to adjust at a different rate, regardless of training prior to the introduction of the system [10]. In 2010, 5.3\% of households in Zimbabwe had a computer [11], however most of these households was concentrated in urban areas. ICTs agglomerate more around places where a power grid, as well as the necessary broadcast and communication networks, are available; in other words, more in urban than in rural areas [10], making ICT use in urban areas socio-cultural norm.

Highly educated individuals use the Internet more often and for more versatile purposes than less educated ones [9]. This means that educational back ground can be another influencing factor of how people get used to ICT applications in the workplace.

When the heuristics of the ePMS are done well the software becomes "usable" to the users, there is easy buy-in to the new ePMS. This study sought to find out whether or not the users of the ePMS felt that its heuristics made it a usable system taking into accounts demographic and socio-cultural differences.

\section{Methods}

A self administered questionnaire was constructed using heuristics concepts from the Agency of Healthcare Research and Quality's “Electronic Health Record Usability-Evaluation and Use Case Frame Work” document. This 5 point likert scale questionnaire was designed to investigate user's perceptions of the electronic patient management system (ePMS) on its overall usability based on the heuristics of the system. Each heuristic aspect had its own section in the questionnaire. Essentially, the questionnaire asked whether or not the respondents agreed that the ePMS is usable. The study was piggybacked on the Ministry of Health and Child Care (MoHCC) AIDS and TB Unit's field work (which involved visiting the 83 high volume health facilities where the system was installed in the first phase of installation). Thus the questionnaire was distributed via AIDS and TB Unit team members who were doing MoHCC field work at those 83 facilities. The piggybacking was done because that is the kind of access that the study was allowed by MoHCC, and also because individually visiting the facilities without making use of the MoHCC teams and transportation resources would have been costly. The AIDS and TB unit ePMS teams interact with the target respondents (who are ePMS users in the following categories: healthcare managers; healthcare providers and data entry clerks) during their field work.

The study was a cross-sectional facility survey, to investigate whether or not the users of the AIDS and TB 
ePMS agreed that it was a usable system based on its heuristics. It was both quantitative and qualitative in nature.

The targeted respondents from which all data for this study came where any healthcare managers, healthcare providers and data entry clerks that had been trained by the ePMS team on the use of the ePMS and were involved in the use of ePMS for provision of AIDS and TB services at healthcare facilities where they worked. In this case the 83 healthcare facilities where ePMS was installed in the $1^{\text {st }}$ phase of ePMS rollout. The questionnaire was reviewed by the AIDS and TB Unit heads of ePMS and piloted in 2 healthcare facilities with ePMS before it was deployed.

The first variable the study looked at was usability. The questionnaire was score and a threshold score was determined (the threshold score was the score that one would get if he/she answered undecided for every question). That score was 93 for the entire questionnaire. Any score above 93 indicated that the respondent agreed that the ePMS was usable. A score of 93 was interpreted as undecided and a score bellow 93, disagreement. Both the numerical score and the usability agreement (or disagreement or undecided) were recorded as variables for every respondent. The highest possible score for the questionnaire was 155.

The questionnaire was also divided into sections of different heuristics, each section had its own threshold score, and the total of the threshold scores for the different sections was the threshold score of the entire questionnaire. The heuristics that the questionnaire covered are; software user interaction, learnability, cognition facilitation, user control and software flexibility, system real-world match, navigation and existing, consistency, defaults, software system interaction, help and documentation, and error management. These were adopted from the Agency for Healthcare Research and Quality "Electronic Health Record Usability: Evaluation and Use Case Frame Work" publication [4].

The other variables that were recorded include: The designation of the respondent, that is, whether he/she was a healthcare manager, a healthcare provider or a data entry clerk; The gender of the respondent was recorded; The age in years, of the respondents was recorded; The period of time the respondent had spent working at that institution was recorded in months and years; The number of years of experience that the respondent had in his/her profession was recorded in years and months; The period of time for which the facility had been using the ePMS was recorded; Exposure of the respondent to electronic health interventions prior to ePMS was also noted; If the respondent had prior exposure to electronic health interventions, the number of years of experience with electronic health interventions was also recorded; The province in which the health facility is situated was also recorded. These variables were recorded because they were thought to potentially influence the user's perception of usability based on the literature review that had been done.

Data was analyzed using Stata ${ }^{\circledR}$ 13. Measures of spread of the numerical score per questionnaire were calculated, the demographics of the respondents were described also, categorical and numerical variables were described. Multiple linear regression analysis to find out if the numerical score (and hence usability) varied with; Age, Gender, "period of time a respondent had worked at a facility", "the respondent's number of years of experience in his/her profession", "the period of time for which the facility had had ePMS" and the respondent's number of years of experience with ehealth interventions if any. The multiple regression analysis was done at 95\% confidence level taking not of the P-value and the t-statistic for each variable being analyzed. This is an appropriate test for all the numerical variables and some categorical variables such as gender.

Pearson's chi squared test and fisher's exact test where done for gender and usability; and for time the facility had had ePMS and usability; age group (at 10 year intervals) and usability; province and usability as well as previous exposure to ehealth interventions and usability. These were appropriate tests for all the categorical variables measured. Fisher's exact test was added because is it appropriate where there are expected values below 5 and the number of observations is low.

The following variables' association with the questionnaire score and/or usability were measured:

Age; Gender ; Period of time a respondent had worked at a facility and the score; The respondent's number of years of experience in his/her profession and the score; The period of time for which the facility had had ePMS and the score; The respondent's number of years of experience with ehealth; The period of time for which the facility had had ePMS; Age group and usability and Province.

Ethics approval and relevant permission were sought and granted before carrying out the study.

\section{Results}

Self administered questionnaires were distributed via the ePMS teams of the AIDS and TB Unit that were on deployment to the 83 health facilities. The questionnaire asked whether or not the users of the ePMS agreed that 
the ePMS was usable. Multiple linear regression analysis was done using Stata ${ }^{\circledR} 13$ for numerical variables and Pearson's chi-squared as well as Fisher's exact tests were done for categorical variables.

48 out of 83 facilities responded with one respondent per responding facility affording this research a study population representation at $95 \%$ confidence level at $10 \%$ confidence interval.

\subsection{Description of Important Variables}

Table 1: Distribution of questionnaire scores from respondents: At $n=48$, the mean score was 113.9167 with a standard deviation of 18.16454. A score bellow 93 was categorised as disagree, a score of 93 as undecided and a score above 93 was categorized as agree in the usability categories.

Table 2: Distribution of usability perception: shows the number of respondents that agreed that ePMS was usable, by virtue of scoring above 93 in their questionnaire responses and those that disagreed by virtue of scoring belong 93 in the questionnaire responses along with those that were undecided by virtue of scoring of 93 .

Table 3: At $n=48$ the mean age was 33.04167 with a standard deviation of 9.773952 .

Table 4 shows the distribution of the respondents by age group where $n=48$.

Table 5 shows the distribution of respondents by gender, $n=48$.

\subsection{Cross Tabulations and Analytical Tests Applied}

Table 6 shows gender against usability where $\mathrm{n}=48$. Pearson chi $^{2}(2)=2.5415 ; \mathrm{P}=0.281$ and Fisher's exact $=$ 0.360 .

Table 7 shows the cross tabulation of respondent's designation and usability. Pearson's chi $^{2}(4)=4.8980=P=$ 0.298 and Fisher's exact $=0.467$.

In Table 8, tEPMS is the period for which a facility has had ePMS. The Pearson chi ${ }^{2}=4.9286$ and $\mathrm{P}=0.085$. Fisher's exact test $=0.07$.

Table 9, $\mathrm{n}=48$; Pearson $\mathrm{chi}^{2}=1.3174$ and $\mathrm{P}=0.504$ and Fisher's exact test $=1.000$.

A multiple linear regression analysis to find out if the numerical score (and hence usability) varied with; Age, Gender, "period of time a respondent had worked at a facility", "the respondent's number of years of experience in his/her profession", "the period of time for which the facility had had ePMS" and the respondent's number of years of experience with ehealth interventions. The results are shown in Table 10: Multiple linear regression analysis (95\% confidence intervals)The confidence level for the analysis was $95 \%$ and $n=48$ for all variables where yrfacilityn = the number of years a respondent had worked at a facility; yrprofn = the respondent's number of years of experience in his/her profession; tEPMS = the time in years and months for which the facility had been on ePMS; yrEHLTH = the number of years of experience (if any) that the respondent had with electronic health interventions. The R-squared value is 0.2623 .

Table 1. Distribution of questionnaire scores from respondents.

\begin{tabular}{|ccc|}
\hline & & Total score \\
\hline Percentiles & & Smallest \\
\hline $\mathbf{1 \%}$ & 70 & 70 \\
$\mathbf{5 \%}$ & 84 & 70 \\
$\mathbf{1 0 \%}$ & 93 & 84 \\
$\mathbf{2 5 \%}$ & 104 & 84 \\
$\mathbf{5 0 \%}$ & 113 & Largest \\
& & 139 \\
$\mathbf{7 5 \%}$ & 128.5 & 139 \\
$\mathbf{9 0} \%$ & 138 & 140 \\
$\mathbf{9 5 \%}$ & 139 & 140 \\
\hline $\mathbf{9 9} \%$ & 140 & \\
\hline
\end{tabular}


Table 2. Distribution of usability perception.

\begin{tabular}{cccc}
\hline Usable agreement level category & Frequency & Relative frequency (\%) & Cumulative relative frequency (\%) \\
\hline Undecided & 2 & 4.17 & 4.17 \\
Agree & 42 & 87.50 & 91.67 \\
Disagree & 4 & 8.33 & 100 \\
Total & 48 & 100 & \\
\hline
\end{tabular}

Table 3. Distribution of respondent's numerical age in years.

\begin{tabular}{|ccc|}
\hline Percentiles & & Age \\
\hline $\mathbf{1 \%}$ & 22 & Smallest \\
$\mathbf{5 \%}$ & 24 & 22 \\
$\mathbf{1 0 \%}$ & 25 & 22 \\
$\mathbf{2 5 \%}$ & 26.5 & 24 \\
$\mathbf{5 0 \%}$ & 30 & 24 \\
& & Largest \\
$\mathbf{7 5 \%}$ & 37 & 57 \\
$\mathbf{9 0 \%}$ & 45 & 57 \\
$\mathbf{9 5 \%}$ & 57 & 60 \\
$\mathbf{9 9 \%}$ & 60 & 60 \\
\hline
\end{tabular}

Table 4. Distribution of respondents' age by age group.

\begin{tabular}{cccc|}
\hline Age group in years & Frequency & Relative frequency (\%) & Cumulative Relative frequency (\%) \\
\hline $\mathbf{2 0}$ to $\mathbf{2 9}$ & 22 & 45.83 & 45.83 \\
$\mathbf{3 0}$ to $\mathbf{3 9}$ & 18 & 37.50 & 83.33 \\
$\mathbf{4 0}$ to $\mathbf{4 9}$ & 4 & 8.33 & 91.67 \\
$\mathbf{5 0}$ to $\mathbf{5 9}$ & 2 & 4.17 & 95.83 \\
$\mathbf{6 0}$ to $\mathbf{6 9}$ & 2 & 4.17 & 100.00 \\
Total & $\mathbf{4 8}$ & $\mathbf{1 0 0}$ & \\
\hline
\end{tabular}

Table 5. Distribution of respondents by gender.

\begin{tabular}{cccc}
\hline Gender & Frequency & Relative frequency (\%) & Cumulative Relative frequency (\%) \\
\hline Male & 22 & 45.83 & 45.83 \\
Female & 26 & 54.17 & 100.00 \\
Total & 48 & 100 & \\
\hline
\end{tabular}

Table 6. Pearson chi ${ }^{2}$ and fisher's exact test between gender and usability (2 degrees of freedom).

\begin{tabular}{ccccc}
\hline & Usability & & Disagree & Total \\
\hline Gender & Undecided & Agree & 2 & 22 \\
Male & 2 & 18 & 1.8 & 22.0 \\
Expected frequency & 0.9 & 19.3 & 2 & 26 \\
Female & 0 & 24 & 2.2 & 26.0 \\
Expected frequency & 1.1 & 22.8 & 4 & 48 \\
Total & 2 & 42 & 4.0 & 48.0 \\
Expected frequency & 2.0 & 42.0 & & \\
\hline
\end{tabular}


Table 7. Pearson chi ${ }^{2}$ and fisher's exacts test for designation of respondent and usability (4 degrees of freedom).

\begin{tabular}{ccccc}
\hline & Usability & & Total \\
\hline Designation & Undecided & Agree & Disagree & 6 \\
\hline Manager & 0 & 6 & 0 & 6.0 \\
Expected frequency & 0.3 & 5.3 & 0.5 & 14 \\
Provider & 0 & 14 & 0 & 14.0 \\
Expected frequency & 0.6 & 12.3 & 1.2 & 28 \\
Data Entry Clerk & 2 & 22 & 4 & 28.0 \\
Expected frequency & 1.2 & 24.5 & 2.3 & 48 \\
Total & 2 & 42 & 4 & 48.0 \\
\hline
\end{tabular}

Table 8. Pearson chi $^{2}$ and fisher's exact tests for period for which the facility has had ePMS and usability (2 degrees of freedom).

\begin{tabular}{ccccc}
\hline & & Usability & & Total \\
\hline tEPMS & Undecided & Agree & Disagree & 32 \\
\hline Less than 1 year & 0 & 30 & 2 & 32.0 \\
Expected frequency & 1.3 & 28.0 & 2.7 & 16 \\
$\mathbf{1}$ to 2 years & 2 & 12 & 1.3 & 16.0 \\
Expected frequency & 0.7 & 14.0 & 4 & 48 \\
Total & 2 & 42 & 4.0 & 48.0 \\
\hline
\end{tabular}

Table 9. Previous exposure to ehealth interventions and usability, Pearson chi ${ }^{2}$ and Fisher’s exact test (2 degrees of freedom).

\begin{tabular}{ccccc}
\hline & & Usability & & \\
\hline Previous exposure to ehealth & Undecided & Agree & Disagree & Total \\
\hline No & 2 & 34 & 4 & 40 \\
Expected frequency & 1.7 & 35.0 & 3.3 & 40.0 \\
Yes & 0 & 8 & 0 & 8 \\
Expected frequency & 0.3 & 7.0 & 4 & 4.0 \\
Total & 2 & 42 & 4.0 & 48.0 \\
Expected frequency & 2.0 & 42.0 & & 48 \\
\hline
\end{tabular}

Table 10. Multiple linear regression analysis (95\% confidence intervals).

\begin{tabular}{ccc}
\hline Variable & t-statistic & -0.16 \\
Age & 0.56 & 0.871 \\
Gender & -0.89 & 0.581 \\
Respondent's time in years at the facility (yrfacilityn) & 0.80 & 0.379 \\
Respondent's years of experience in profession (yrprofn) & -3.20 \\
Period of time in years the facility has been on ePMS (tEPMS) & 0.52 \\
Respondent's years of experience with electronic health interventions.
\end{tabular}




\section{Discussion}

The possible factors that could influence the users' perceptions of the electronic patient management system (ePMS), for AIDS and TB service in Zimbabwe, were analyzed. The analysis of some of these factors, especially age and gender was inspired by the studies and findings of M. Geldof and R. Holloway and well as M. Nasi and colleagues. Though these studies were looking at how response to information communication technologies (ICTs) (and not particularly ehealth applications such as ePMS), varies with age, gender and other socioeconomic factors, and in particular M. Nasi and colleagues were looking at online communities. Reference can still be made to these studies since ePMS is essentially ICT in the healthcare setting and the same principles should apply.

The P-value for the chi ${ }^{2}$ test at 2 degrees of freedom for the effect of gender on usability is 0.281 as shown in Table 6: Pearson's chi ${ }^{2}$ and Fisher's exact test between gender and Usability ( 2 degrees of freedom). This implies that any differences between the 2 genders are by chance and there is no signuficant difference between the 2 genders in their perception of the usability of the ePMS. A difference would have been expected owing to gender inequalities and disparities in education and ICT use in Africa [10]. However, in this study, it was not so. This could be because the training on the use of the ePMS that was given to the users by the ePMS team and heads was good enough to cancel out that "gender divide". Part of this gender effect includes the education (literacy) gap found between males and females in some countries where women are marginalized. However with a 2011 male literacy rate of $87.8 \%$ and a female rate of 80.1\% [12] such gaps are highly unlikely in Zimbabwe, especially in the formal employment sector. However, it is important to bear in mind that this finding is based on the response of 48 out of the 83 facilities. An alternative test, Fisher's exact test had a P-value of 0.360 , this is confirmatory of the chi ${ }^{2}$ test findings.

Table 7: Pearson's chi ${ }^{2}$ and Fisher's exacts test for designation of respondent and Usability; shows a chi ${ }^{2}$ test and Fisher's exact tests that were done to assess differences in the designations (posts) of the healthcare workers' perceptions on the usability of the ePMS system. The chi ${ }^{2} \mathrm{P}$-value is 0.298 showing no difference. The Fisher's exact test of 0.467 is confirmatory. This is a rather interesting finding. The 3 different designations of the targeted respondents were healthcare manager, healthcare provider and data entry clerk. The data entry clerks are supposedly people that have an ICT educational back ground and are employed by the AIDS and TB unit to assist with operating the ePMS, chiefly their role is to enter data retrospectively, while the healthcare managers and healthcare providers and managers feed current (real time) data into the ePMS. None of the data entry clerks had been working at any of the health facilities for more than 2 years when the study was done. They essentially came together with, and were necessitated by the ePMS. They are expected to find the ePMS more usable compared to the 2 other groups, even if heuristic of the ePMS system were 'bad', simply because of their ICT educational background [10]. Again the lack in difference may be attributed the training that was given by the ePMS heads. Healthcare managers, healthcare providers and data entry clerks received the same ePMS training. This implies again that the training may have been good enough to cancel out the "educational (ICT savvy) divide". Otherwise it implies that the heuristics of the system are good from the perspective of the users and the system is indeed usable.

In Table 9 Previous exposure to ehealth interventions is not sufficient enough to create a difference in, because not all ehealth applications are the same or work the same way. Mobile health and ePMS, for example, are completely different. Having exposure (and experience) in one may not give proficiency in the other.

In the multiple linear regression analysis to find out if the numerical score (and hence usability) varied with; Age, Gender, "period of time a respondent had worked at a facility", "the respondent's number of years of experience in his/her profession", "the period of time for which the facility had had ePMS" and the respondent's number of years of experience with ehealth interventions shown in Table 10: Multiple linear regression analysis (95\% confidence intervals). Usability varies with none of the variables but one. Based on M. Geldof and R. Holloway and M. Näsi, P. Räsänen, and V. Lehdonvirta's opinions, these are unlikely findings. In this analysis, the one variable that usability varies with is "the period of time for which the facility had had ePMS". The variable has a P-value of 0.003 which shows significance. The Pearson chi ${ }^{2}$ and Fisher's exact test for this variable (see Table 8: Pearson's chi ${ }^{2}$ and Fisher exact tests for period for which the facility has had ePMS and usability) have P-values of 0.085 and 0.07 respectively. These are almost significant. This implies that the more time the healthcare works at a facility spend with the ePMS the better their perception of its usability (and heuristics) becomes. While this is good, this is also interesting because it is difficult to tell if that improvement in usability actually has anything to do with improved heuristics, which are the foundation of the usability assessment in the 
first place. It could be that the more time they spend with the system, the better accustomed they become to it, hence they become moreable to go around the unchanged heuristics of the system and find it more and more usable with time.

\subsection{Bias}

The biases that questionnaires are prone to cannot be ruled out. These are; central tendency bias where respondents avoid using extreme response categories, acquiescence response bias where respondents tended to agree with statements as they were presented and social desirability bias where respondents could have tried to portray their health facility and department in a good light.

Also, considering that the questionnaires were sent to respondents through the AIDS and TB Unit ePMS team and that the study was targeting 83 healthcare facilities and respondents from only 48 facilities were "willing" to participate. These factors coupled with the fact that $87.5 \%$ of the respondents agreed that the system was usable. It is possible that the ePMS team on deployment by the AIDS and TB unit of MoHCC targeted healthcare facilities where they anticipated favourable results; since ePMS is their project. Otherwise the results imply that the heuristic of the system are good from the perspective of the users and the system is indeed usable.

\subsection{Limitations}

A shortcoming of the study is the number of respondents per facility. It would have been ideal to get at least 1 healthcare manager, 1 healthcare provider and 1 data entry clerk, respond and from each and every one of the 83 targeted facilities as was the initial plan (as opposed to one responded per healthcare facility from only 48 of the facilities). This would have produced high quality reliable data compared to what the study actually got. However, relying on the resources and the of the AIDS and TB unit, made this impossible.

The study was also unique in that heuristics test should normally be done by ICT experts looking at the system (expert diagnosis) and making their conclusions [4]. This study brought a twist, where the perceptions of the users pertaining to the heuristics and usability of the system were put in the forefront. At the end of the day it is the users that spend more time with these electronic health applications such as ePMS, not "passerby" experts.

\section{Conclusions and Recommendations}

The questionnaire was an applicable tool for the usability assessment, though it could have been augmented with experts performing diagnostics on the system and drawing conclusions on the heuristics and usability of the system as it recommended by the Agency for Healthcare Research and Quality.

While other associations could not be found, usability of the ePMS from the user's perspective improves with time. The longer the users spend with the ePMS system, better its usability becomes.

If the Ministry of Health and Child Care through the AIDS and TB unit is to maximise benefits from the use of their ePMS system, it is important that they among other things continue to acquire information to do with user perceptions and experiences. Doing this study in a way that will curb its limitations will produce reliable results of good quality. This includes getting response from all the facilities involved and having all categories of relevant healthcare workers (healthcare managers, healthcare providers and data entry clerks in this scenario) from each of the relevant healthcare facilities participating in the study.

The issue of user perceptions of newly installed electronic health (ehealth) applications such as ePMS inevitably leads to issues of change management. Since this is a new ehealth intervention in the Zimbabwean setting, it is important for MoHCC to look into possible gaps in its change management strategy, before it proceeds to the next phases of ePMS rollout. Better the planning leads to better chances of success.

\section{Acknowledgements}

This work was supported by the National Institute of Health Fogarty International Centre (Grant Number: 4D43TW007004-13).

\section{Key Definitions}

Usability: The degree to which a user of an electronic (health) application or intervention, software or system 
finds it easy to use.

Users: The people that use the electronic patient management system to provide AIDS and TB services at the health facilities through Zimbabwe. They fall into categories: healthcare managers, healthcare providers and data entry clerks.

\section{References}

[1] AIDS \& TB Unit Ministry of Health and Child Care (2012) Zimbabwe Rapid Assessment: A Roadmap for the Electronic Patient Management System for HIV/TB.

[2] Nielsen Norman Group. Usability 101: Introduction to Usability. http://www.nngroup.com/articles/usability-101-introduction-to-usability/

[3] Nielsen Norman Group. 10 Heuristics for User Interface Design: Article by Jakob Nielsen. http://www.nngroup.com/articles/ten-usability-heuristics/

[4] Agency for Healthcare Research and Quality (2009) Electronic Health Record Usability, Evaluation and Use Case Framework. Electronic Health Record Usability, 09.

[5] McLean, V. (2006) Electronic Health Records Overview. MITRE Corparation. National Institutes of Health. National Center for Research Resources. April, 2006.

[6] Maine Health Info Net (2011) Value of an EHR after Hurricane Katrina. Youtube, United States of America.

[7] Agency for Healthcare Research and Quality (2009) Electronic Health Record Usability: Interface Design Considerations. Electronic Health. Record Usability, 09.

[8] Gale, L. (1999) Graphics and Web Design Based on Edward Tufte’s Principles.

[9] Näsi, M., Räsänen, P. and Lehdonvirta, V. (2011) Identification with Online and Offline Communities: Understanding ICT Disparities in Finland. Technology in Society, 33, 4-11. http://dx.doi.org/10.1016/j.techsoc.2011.03.003

[10] Geldof, M. and Holloway, R. (2011) Earphones Are Not for Women : Gendered ICT Use among Youths in Ethiopia and Malawi. Information Technologies \& International Development, 7, 69-80.

[11] Techzim. ICT Development Index: Zimbabwe Moves Four Places up | Techzim. www.techzim.co.zw/2011/09/ict-development-index-zimbabwe-moves-four-places-up/

[12] Index Mundi (2014) Zimbabwe Literacy—Demographics. http://www.indexmundi.com/zimbabwe/literacy.html

\section{Acronyms and Abbreviations}

AIDS: Acquired Immune Deficiency Syndrome;

Ehealth: Electronic health;

EMR: Electronic Medical Records;

ePMS: Electronic patient management system;

HIV: Human immunodeficiency virus;

ICT: Information communication technology;

MoHCC: Ministry of Health and Child Care;

TB: Tuberculosis. 